\title{
3D Blood Vessels Reconstruction Based on Segmented CT Data for Further Simulations of Hemodynamic in Human Artery Branches
}

\author{
Andrzej POLAŃCZYK ${ }^{1}$, Michał STRZELECKI ${ }^{2}$, Tomasz WOŹNIAK ${ }^{2}$, \\ Wojciech SZUBERT ${ }^{3}$, Ludomir STEFAŃCZYK ${ }^{3}$
}

\begin{abstract}
We aimed at the reconstruction of the branches of human aortic arch for blood perfusion analysis used later in the Computational Fluid Dynamic (CFD). The reconstruction was performed based on segmentation results obtained from CT data. Two segmentation algorithms, region growing and level set were implemented. Obtained binary segmentation results were next evaluated by the expert and corrected if needed. The final reconstruction was used for preparation of a numerical grid and for further calculation of blood hemodynamic. The collected data composed of blood velocity and blood flow rate in function of time were compared with USG-Doppler data. Results demonstrate that proposed algorithm may be useful for initial reconstruction of human cardiac system, however its accuracy needs to be improved as further manual corrections are still needed.
\end{abstract}

Keywords: vessel tree segmentation and modelling, blood flow simulation, 3D data reconstruction, CFD technique

\section{Introduction}

Current medical imaging techniques enable detailed visualization of human vasculature thank to computed tomography (CTAngio) or magnetic resonance (MRI) angiographic sequences [21]. This 3D data allows building accurate numerical vessel tree models that have numerous medical applications, e.g. are very useful for surgery planning [11]. Moreover, this approach may be treated as a core for blood flow simulation that results in estimation of blood volume and pressure in various fragments of vascular network (located e.g. inside of the crane) where these parameters cannot be measured by routine methods like Doppler ultrasound. Blood flow simulation provides important information, crucial for assessment of blood distribution for patients affected by vascular diseases, e.g. stenosis or arterial tortuosity syndrome. Numerical simulation enables evaluation of how implanting of stents or vascular grafts inside the artery

${ }^{1}$ Department of Heat and Mass Transfer, Lodz University of Technology, Wólczańska 213, 90-924 Łódź, Poland

${ }^{2}$ Lodz University of Technology, Wólczańska 211/215, 90-924 Łódź, Poland

3 Department of Diagnostic Imaging Medical University of Lodz, Kopcińskiego 22, 90-154 Łódź, Poland 
is affecting a blood flow in reconstructed vessel. Moreover, blood flow simulation can be used as a verification technique for perfusion assessment in MR perfusion-weighted imaging, since there is a lack of standardized and optimized perfusion MRI protocols and lack of straightforward guidelines on how to interpret measurement results [18].

The use of numerical methods e.g. Computational Fluid Dynamic (CFD) technique in solving problems related to blood flow in cardiac system is widely described in the literature $[10,19]$. CFD technique is most often performed with the use of the medical data from CTAngio, MRI or X-Ray [1]. However, when the character of the blood flow is obligatory Doppler-US examination is applied [4]. Depending on the type of analyzed hemodynamic in a cardiac system, blood may be treated as a Newtonian $[6,8]$ or non-Newtonian fluid $[3,20]$.

The aim of the study was to verify proposed algorithm of automatic reconstruction of the branches of human aortic arch for blood perfusion analysis, for further use in Computational Fluid Dynamic (CFD).

\section{Materials and methods}

CTAngio data from a female patient age 68, who underwent treatment in the Barlicki Hospital No. 2 in Lodz (Poland) was used in this study. The study protocol was approved by the local ethics committee (approval no.: RNN/126/07/KE). Medical data contained a part of cardiac system from the aortic arch up to brain arteries. Its resolution was 512x512 with 472 slices with voxel size of $0.44 \times 0.44 \times 0.63 \mathrm{~mm} 3$ (Light Speed, GE Medical System). Model of such an image build with the use Light Speed CT visualization software was shown in Figure 1.

Before segmentation algorithms were applied, the CT scans had to be manually adjusted first for brightness, to achieve the highest contrast between blood vessels and surrounding tissues. Then, the first segmentation approach based on region growing technique [7] to extract vessels from the background was implemented. Even after preprocessing (global manual brightness correction), blood vessels brightness varies along $\mathrm{Z}$ - axis therefore, the image must be split into several fragments along this axis. To make such a division the brightness of blood vessels in the image was analyzed. Fragments of the image, where vessels brightness was similar, were separated and analyzed independently. In this particular case, the image was split into 11 segments. Then, the seeds to initialize region growing were established based on the former vessel brightness analysis. Moreover, the region growing brightness range was set. After performing region growing step, all 11 extracted vessel fragments were merged. The algorithm-reconstructed fragment had small gaps in the blood vessels due to large intensity variation inside the vessel. These gaps were eliminated manually using the ImageJ software and its tool for morphological holes filling. 


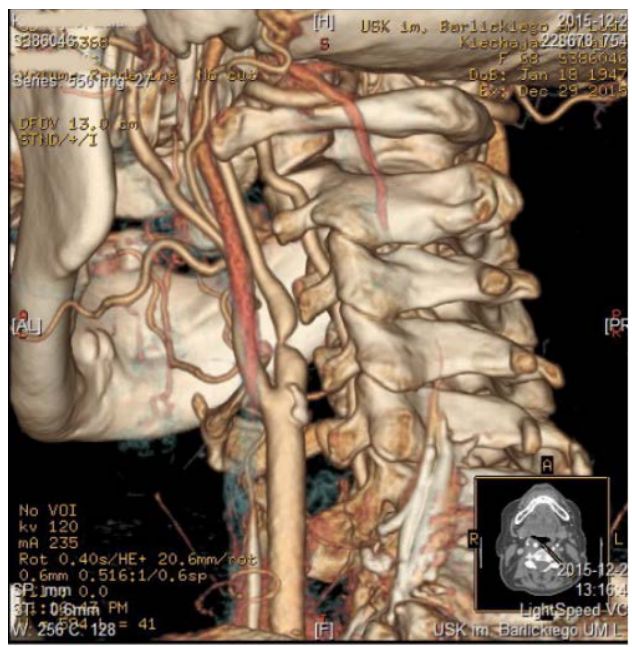

Figure 1. Model of the analyzed fragment of the CT image

The second algorithm used for vascular tree extraction was a level set approach. It is based on mathematical modeling of the image, e.g. through fitting parameterized hypersurfaces to the shape of veins or arteries. The interest in level-set based segmentation algorithms has grown recently, because of relative high speed of these algorithms execution and the method ability of accurate modeling of complex shapes of the blood vessels. These methods are often used as one of the stages of the segmentation procedure [16]. As a levelset function Chan-Vese mathematical model [2] was implemented, as it is widely used for blood vessel extraction. the Euler-Lagrange equation for a Chan-Vese level set evolution is given by:

$$
\frac{\partial \Phi}{\partial \mathrm{t}}=\delta(\Phi)\left[\mu \cdot \operatorname{div}\left(\frac{\nabla \Phi}{|\nabla \Phi|}\right)-\mathrm{b}_{1}\left(\mathrm{VF}(\mathrm{x})-\mathrm{c}_{1}\right)+\mathrm{b}_{2}\left(\mathrm{VF}(\mathrm{x})-\mathrm{c}_{2}\right)\right]
$$

where $\Phi$ denotes the curve contour that evolves in time to fit to the vessels, $\delta(\Phi)$ is a derivative of a Heaviside function $\mathrm{H}(\Phi(x)), \operatorname{VF}(x)$ is the value of a vesselness function (see explanation below) calculated for image point at spatial coordinates $x=\left(x_{1}, x_{2}, x_{3}\right), c_{1}$ and $c_{2}$ are means of inner and outer regions (denoting vessel fragments and other tissues respectively) of a level set function, finally $b_{1}, b_{2}$, and $\mu$ are numerical constants of the equation,

Prior to the segmentation, Hessian filtration [15] was performed as a base for estimation of vesselness function (VF) [5]. The purpose of vesselness function is to enhance vessel structures with eventual goal of vessel segmentation [21]. The vessel enhancement is a filtering process that searches tubular structures in image. The detection of vessel is based on analysis of second order information (Hessian) obtained by image convolution with second order Gaussian derivatives. The scale in this filtration is established by parameter $s$ in Gaussian derivative equation. Hessian matrix is calculated for every voxel in the three-dimensional image. Comparison of eigenvalues of this Hessian matrix to certain values is used for detection 
of tubular structure. The vesselness function equation for one scale $s$ is presented in [5]. In this study, the scale $s$ varied from 2 (brain arteries) to 6 (fragments of aorta). Finally, the LS segmentation is performed on preprocessed images, thus it is applied to three-dimensional VF estimated for the CT cross-sections [22].

3D reconstructions with the use of semiautomatic algorithms happened to have gaps and not continuous structure. Therefore, the third algorithm- the commercial one (3DDOCTOR software (Able Software Corp., Lexington, MA, USA)) was used. The resulted binary vessels tree fragments were used to build 3D virtual models (Figure 2) with the use of a 3DDOCTOR software (Able Software Corp., Lexington, MA, USA). As the reconstructed arteries had still gaps and noises (Figure 2a, b) it was necessary to correct the 3D models manually to receive a shape used in CFD analysis (Figure 2c).

a)

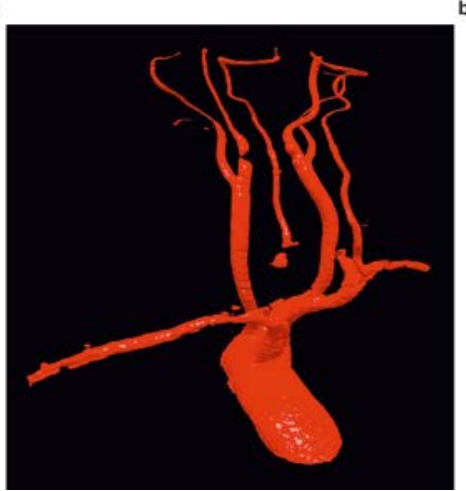

b)

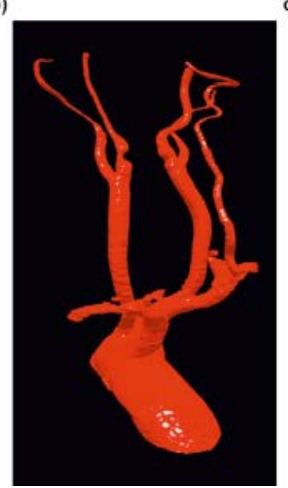

c)

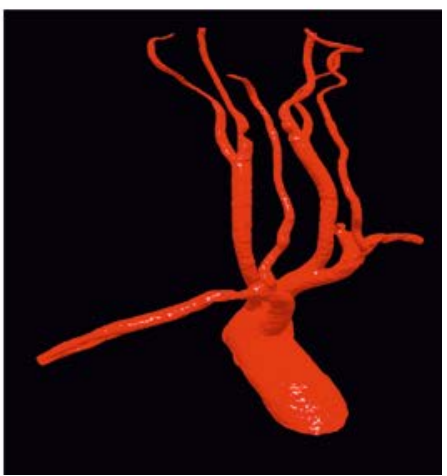

Figure 2. 3D geometries of analyzed part of circulatory system: a) reconstructed with the first homemade algorithm $b$ ) the second homemade algorithm and c) reconstructed with commercial algorithm of 3DDoctor software

Next, CFD technique was applied for blood hemodynamic reconstruction. In the first step, the pre-processor GAMBIT 2.2.30 software (ANSYS Inc., Canonsburg, PA, USA) was used to generate (Figure $3 a$ ) and discretize three-dimensional numerical geometry (Figure $3 b$ ).

a)

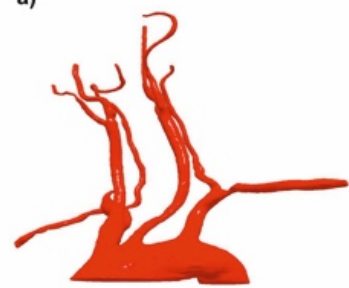

b)

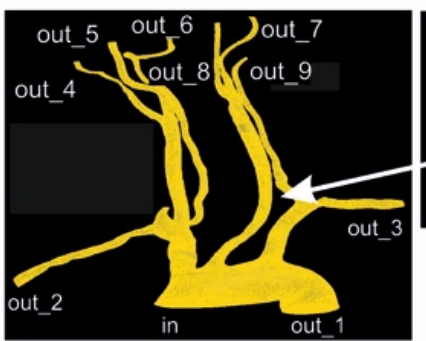

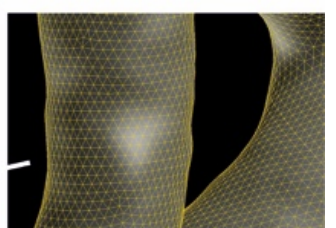

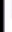

Figure 3. Part of an analyzed cardiac system: 3D geometry preliminary reconstructed by the algorithm and corrected manually (a), numerical grid generated for the analyzed 3D geometry (b) 
Prepared numerical grid was composed of approximately 2,000,000 tetrahedral elements, with boundary layer next to the wall and underwent mesh independent test to neglect the influence of the size or number of numerical grid elements on the results of computer simulation. Next, numerical calculation of blood hemodynamic in the analyzed domain was carried out with the use of ANSYS FLUENT.12.1 software (ANSYS, USA), using Navier Stokes equations (eq. 2 and 3), as previously described in [14].

$$
\nabla \cdot u=0
$$

where:

$u$ - fluid velocity $\left[\mathrm{m} \mathrm{s}^{-1}\right]$

$$
\rho \cdot\left(\frac{\partial u}{\partial t}+u \cdot \nabla u\right)=F-\nabla p+\mu \cdot \Delta u
$$

where:

$\rho$ - fluid density, $\left[\mathrm{kg} \mathrm{m}^{-3}\right]$

$F$ - external forces, $[\mathrm{Pa}]$

$p$ - pressure, $[\mathrm{Pa}]$

Three boundary conditions were applied: (1) at the inlet to the geometry velocity-inlet $(\mathbf{v}(x, y, z)),(2)$ at the outlets from the geometry the boundary condition $\mathrm{p}=$ const. was used, (3) and wall was treated as a rigid structure. Blood was treated as a non-Newtonian liquid with constant density value (equal to $1040 \mathrm{~kg} \mathrm{~m}^{3}$ ) [13]. Rheological properties of a blood were described with the use of Quemada's model (eq. 4 and 5), as previously described in [12]. Blood hematocrit (Htc) was the same as measured in the patient and set at $40 \%$ level.

$$
\eta=\eta_{p} \cdot\left(1-\frac{K \cdot H t c}{2}\right)^{-2}
$$

where:

$\eta_{p}$ - plasma viscosity, [Pa s]

$K$ - inner viscosity of erythrocyte (eq. 4), [-]

Htc - hematocrit, [-] 


$$
K=\frac{k_{0}+k_{\infty} \cdot\left(\frac{\gamma}{\gamma^{c}}\right)^{\frac{1}{2}}}{1+\left(\frac{\gamma}{\gamma^{c}}\right)^{\frac{1}{2}}}
$$

where:

$k_{0}, k_{\infty}$ - parameters which describes blood character, [-]

$\gamma$ - shear rate value, $\left[\mathrm{s}^{-1}\right]$

$\gamma c$ - critical shear rate value, $\left[\mathrm{s}^{-1}\right]$

Moreover, USG-Doppler (GE Vivid 7, GE Healthcare, USA) examination from an examined patient at different levels (Fig.3) was applied. First, all USG profiles were analyzed to extract velocity profiles as a function of time including one whole cardiac cycle. After that ten velocity profiles were prepared, one as an inlet boundary and nine for the verification of outlet conditions.

\section{Results and Discussion}

We analyzed two semiautomatic algorithms for the reconstruction of a part of circulatory system starting from aortic arch up to carotid arteries. As a reference one 3D geometry reconstructed with the use of a commercial algorithm (3DDoctor) was applied.

\subsection{Spatial configuration analysis}

We analyzed three geometries reconstructed with the use of three different image processing algorithms. First two 3D models which were reconstructed with the use of semiautomatic algorithms which happened to have gaps and not continuous structure. While, the third algorithm- the commercial one- had continuous structure without gaps and fake wall shape (Figure 4c). The first homemade algorithm reconstructed 3d structure of analyzed part of circulatory system as 19 separated volumes. Only left external carotid artery was continuous structure. For both subclavian arteries, numerous of gaps appeared (Figure 4a). Moreover, the reconstructed structure was irregular in shape and it did not reflect the real shape of artery's wall. Due to low accuracy of the first algorithm and creation of 19 volumes it also reconstructed incorrectly false ducts inside the true lumen.

Modification of the first algorithm allowed to decrease the number of calculated volumes from 19 to 2 which reminded us still with discrete structure of the analyzed object. Moreover, the left internal carotid artery was not recognized, therefore this part did not appear in analyzed 
3D model. Similarly, to the first algorithm numerous of gaps appeared after reconstruction with the use of the second algorithm. The second algorithm reconstructed properly the length of subclavian arteries $(260 \mathrm{~mm}$ and $140 \mathrm{~mm}$ for the reconstruction with the first and the second algorithm, respectively) however without reflecting its shape (Figure 4b).
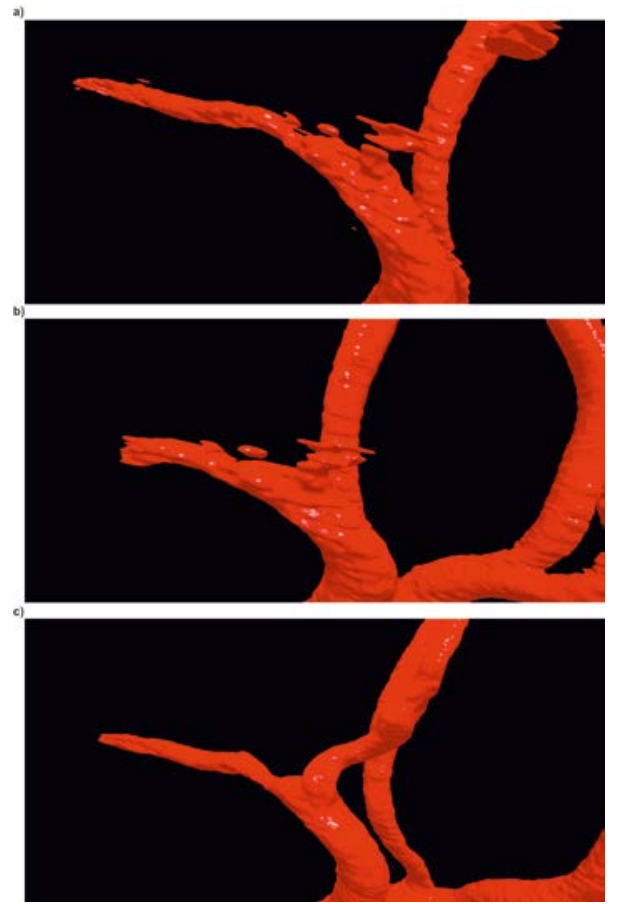

Figure 4. The subclacian artery: a) reconstructed with the first algorithm with numerous gaps and artifacts b) reconstructed with the second algorithm with numerous gaps c) proper duct reconstructed with 3DDoctor

Analysis of spatial configuration of reconstructed geometries was based on calculated volume and surface of the object. The first parameter indicated $10 \%$ and $1.7 \%$ decrease of measured volume for the geometry prepared with the use of first and second semiautomatic algorithm, respectively compared to the 3DDoctor results (Table 1). Moreover, we noticed that the higher the number of artificially created volumes the lower the calculated volume.

The second analyzed parameter, surface for the geometry reconstructed with the first semiautomatic algorithm indicated the highest calculated surface $\left(118874.63 \mathrm{~mm}^{2}\right)$. Comparison of the models reconstructed with the use of first semiautomatic algorithm with 3DDoctor indicated 2.2\% difference (Table 1). While, for the second semiautomatic algorithm and 3DDoctor 2.8\% difference was observed (Table 1). Additionally, comparison of the first and the second algorithm indicated 5.2\% difference in calculated surface for analyzed geometries. 
Table 1. Geometric description of analyzed geometries for the first and second algorithm and commercial algorithm (3DDoctor). Surface measured in $\left[\mathrm{mm}^{2}\right]$ and volume measured in $\left[\mathrm{mm}^{3}\right]$.

\begin{tabular}{|c|c|c|}
\hline Name & Surface $\left[\mathbf{m m}^{2}\right]$ & Volume $\left[\mathbf{m m}^{\mathbf{3}}\right]$ \\
\hline $\begin{array}{c}\text { Semiautomatic } \\
\text { algorithm ver. 1 }\end{array}$ & 118874.63 & 661854.35 \\
\hline $\begin{array}{c}\text { Semiautomatic } \\
\text { algorithm ver. 2 }\end{array}$ & 116265.37 & 719956.53 \\
\hline 3DDoctor & 112999.82 & 740306.06 \\
\hline
\end{tabular}

\subsection{Blood hemodynamic analysis}

Due to low accuracy of both semiautomatic algorithms we used 3DDoctor algorithm for further hemodynamic analysis. Both, blood velocity and blood flow rate in function of time were confronted with USG-Doppler data.

Analysis of blood velocity distribution indicated areas with higher and lower velocity values. This phenomenon was correlated with different areas of outlets and different pressure values set at outlets. Figure 5 represents velocity contours for the time step 0.2s.

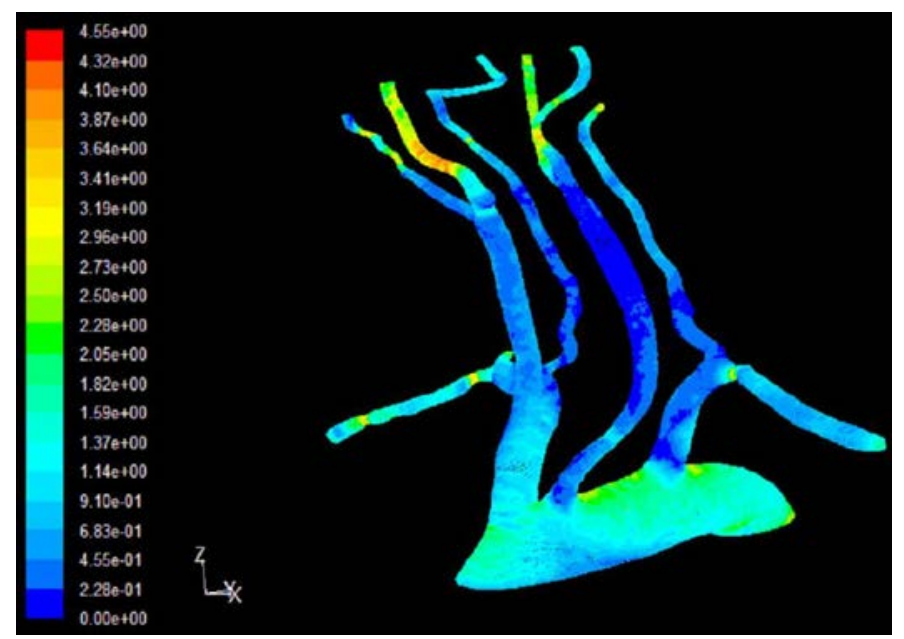

Figure 5. Velocity contours for 3D geometry from CFD calculation for the time step $0.2 \mathrm{~s}$

Quality analysis of velocity profiles in function of time for CFD simulation and DopplerUS examination indicated similar character (Figure 6). 


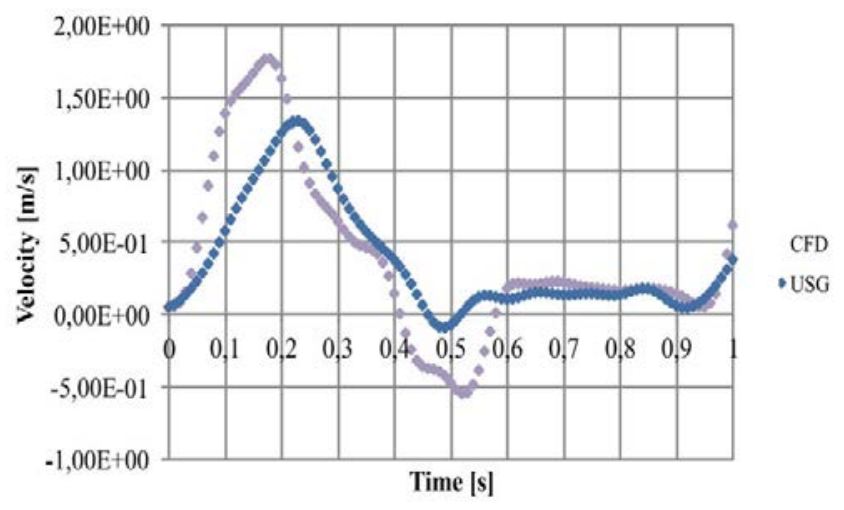

Figure 6. Velocity profile in function of time for CFD and Doppler-US examination

However, the quantitative analysis indicated that for some branches data from CFD and USG markedly vary. For instance, Figure 7 represents data for outlet out_9 where difference in velocity value was about $372.96 \%$ for CFD vs. USG.

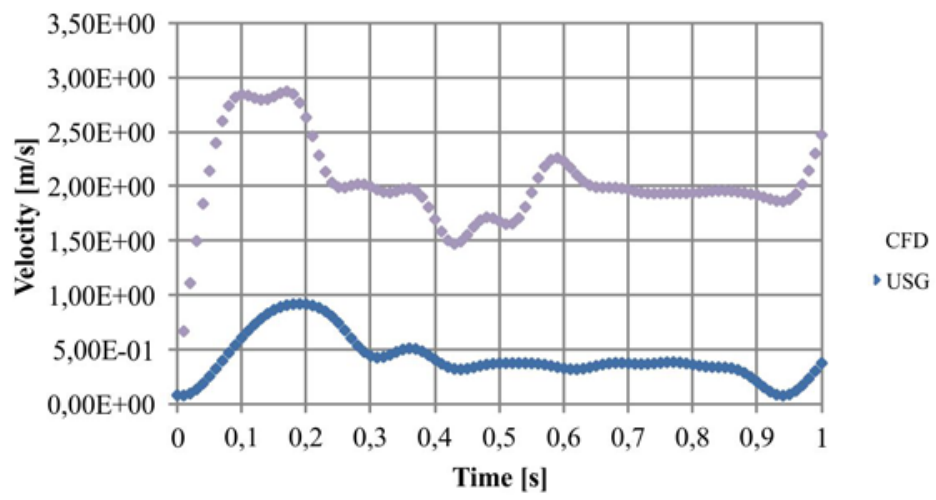

Figure 7. The example of velocity profile in function of time for CFD and Doppler-US examination of out_9

The observed difference might derive from a fact that the rigid wall boundary was set in CFD calculation while the vessel is an elastic structure. Moreover, pressure values at outlets were set as average values, meanwhile in real cardiac system, value of this parameter is considered as a function of time. However, it should be stressed that the highest differences 
between CFD and Doppler-US methods, were observed in narrow vessels with a turbulent character of flow, like the left vertebral artery or the left narrowed internal carotid artery.

CFD simulation indicated that total volume of blood $126.84 \mathrm{ml} \mathrm{s}^{-1}$ was directed into aortic arch where $98.23 \mathrm{ml} \mathrm{s}^{-1}$ (77.44\%) went into abdominal aorta and $28.62 \mathrm{ml} \mathrm{s}^{-1}$ (22.56\%) went into three branches rising from aortic arch (Table 2). CFD analysis of perfusion for the three branches indicated that the highest flow rate, $19.18 \mathrm{ml} \mathrm{s}^{-1}(15.12 \%)$ of the total blood flow, was for the branch where four outlets (out_2, out_4, out_5 and out_6) were placed. The middle branch composed of two outlets (outl_7 and out_8) comprised of $5.33 \mathrm{ml} \mathrm{s}^{-1}$ (4.21\%) of the total blood flow. The third branch composed of two outlets (out_3 and out_9) comprised of $4.10 \mathrm{ml} \mathrm{s}^{-1}$ (3.23\%) of the total blood flow. According to the CFD results the highest flow rate was observed for the internal carotid artery (out_6), $10.87 \mathrm{ml} \mathrm{s}^{-1}$ and the lowest for the external carotid (out_7) $1.30 \mathrm{ml} \mathrm{s}^{-1}$. Hence, calculations for the branch with highest flow rate overlaps.

Next, we confirmed a proposed algorithm of reconstruction together with the use of CFD technique for outlet positions, indicated in Table 2, with the data recorded during Doppler-US examination. Analysis of the perfusion for CFD and Doppler-US data indicated differences from $3.03 \%$ up to $372.96 \%$. Total amount of blood at outlets from CFD model and DopplerUS measurements was similar and amount equal to $126.84 \mathrm{ml} \mathrm{s}^{-1}$ and $114.89 \mathrm{ml} \mathrm{s}^{-1}(-10.40 \%)$, respectively. The smallest difference was observed for out_1 where, the flow was $95.34 \mathrm{ml} \mathrm{s}^{-}$ ${ }^{1}$ and $98.23 \mathrm{ml} \mathrm{s}^{-1}$ (CFD vs. USG). The highest difference was observed for out_9 where, the flow was $0.42 \mathrm{ml} \mathrm{s}^{-1}$ and $2.00 \mathrm{ml} \mathrm{s}^{-1}$ (CFD vs. USG).

Table 2. Blood perfusion in the reconstructed branches for the CFD technique and gathered during the USG-Doppler examination.

\begin{tabular}{|c|c|c|c|}
\hline Number & $\begin{array}{c}\text { Blood flow rate } \\
\text { [ml-s } \mathbf{-}^{-1} \text { ] } \\
\text { USG-Doppler }\end{array}$ & $\begin{array}{c}\text { Blood flow rate } \\
\text { [ml-s }{ }^{-1} \text { ] } \\
\text { CFD }\end{array}$ & $\begin{array}{c}\text { Difference } \\
{[\%]}\end{array}$ \\
\hline in & $114.89(100 \%)$ & $126,84(100 \%)$ & 0 \\
\hline out_1 & $95.34(82.98 \%)$ & $98.23(77.44 \%)$ & -3.03 \\
\hline out_2 & $4,98(4.33 \%)$ & $3.58(2.82 \%)$ & 28.16 \\
\hline out_3 & $2.03(1.77 \%)$ & $2.10(1.66 \%)$ & -3.29 \\
\hline out_4 & $0.86(0.75 \%)$ & $1.34(1.06 \%)$ & -54.90 \\
\hline out_5 & $7.58(6.60 \%)$ & $12.97(10.22 \%)$ & $-71.02 \%$ \\
\hline out_6 & $1.05(0.91 \%)$ & $1.30(1.02 \%)$ & -23.54 \\
\hline out_7 & $1.13(0.98 \%)$ & $1.60(1.26 \%)$ & -41.26 \\
\hline out_8 & $1.49(1.30 \%)$ & $3.74(2.95 \%)$ & -150.69 \\
\hline out_9 & $0.42(0.37 \%)$ & $2.00(1.58 \%)$ & -372.96 \\
\hline
\end{tabular}


Finally, we analyzed blood perfusion in the aortic branches that leads blood to the cranium. Those branches were: out_5 (right internal carotid), out_6 (right vertebral), out_8 (left internal carotid) and, out_9 (left vertebral). The flow for blood perfusion in the cranium was $20.01 \mathrm{ml}$ $\mathrm{s}^{-1}$ and $10.55 \mathrm{ml} \mathrm{s}^{-1}$ for CFD analysis and USG data, respectively. Moreover, analysis of the left and right part of brain separately indicated unequal perfusion in CFD and USG data. CFD results were in line with tendencies observed in USG data. For instance, CFD results indicated that distribution of blood was 5 times higher in the right part comparing to the left part. Similarly, in USG data blood volume was 6 times higher in the right part comparing to the left part. The difference observed in the CTAngio derived from the collateral flow in the artery brain wheel.

\section{Conclusions}

Analyzed 3D data allows building accurate numerical vessel tree models which may be further use for the calculation of blood flow in the vascular system. The proposed home-made algorithms could reconstruct the analyzed fragments of circulatory system; however, the reconstructed objects were not linear and had gaps and artificial ducts. In the current state 3D structures reconstructed by the proposed algorithm are not suitable for CFD calculations. Therefore, for the presentation of numerical reconstruction of blood hemodynamic, 3D geometry of a part of circulatory system with the use of commercial algorithm was made. This approach allowed to reconstruct blood flow from aortic arch up to cranium.

The proposed algorithm may be useful for initial reconstruction of human cardiac system; however, its accuracy needs to be improved as further manual corrections are still needed. In the future, simulated MR angiography [9] can be used for further validation of the reconstruction algorithm and its accuracy.

\section{References}

[1] Auer M., Gasser T.C., Reconstruction and finite element mesh generation of abdominal aortic aneurysms from computerized tomography angiography data with minimal user interactions, IEEE Transactions on Medical Imaging, 29, 2010, 1022-1028.

[2] Chan T.F., Vese L.A., Active Contours Without Edges, IEEE Transaction on Image Processing, 10, 2001, 266-277.

[3] Cloutier G., Zimmer A., Yu F.T., Chiasson J.L., Increased shear rate resistance and fastest kinetics of erythrocyte aggregation in diabetes measured with ultrasound, Diabetes care, 31, 2008, 1400-1402.

[4] Dasari P., Venkatesan B., Thyagarajan C., Balan S., Expectant and medical management of placenta increta in a primiparous woman presenting with postpartum haemorrhage: The role of Imaging, Journal of Radiology Case Reports, 4, 2010, 32-40.

[5] Frangi F., Niessen W.J., Vincken K. L., Viergever M. A., Muliscale Vessel Enhancement Filtering, in: Proc. of MICCAI 1998, 1998, 130-137. 
[6] Gijsen F.J., van de Vosse F.N., Janssen J.D., The influence of the non-Newtonian properties of blood on the flow in large arteries: steady flow in a carotid bifurcation model, Journal of Biomechanics, 32, 1999, 601-608.

[7] Gonzales R., Woods R., Digital Image Processing, Addison-Wesley, 1983.

[8] Hoi Y., Meng H., Woodward S.H., Bendok B.R., Hanel R.A., Guterman L.R., Hopkins L.N., Effects of arterial geometry on aneurysm growth: three-dimensional computational fluid dynamics study, Journal of Neurosurgery, 101, 2004, 676-681.

[9] Klepaczko A., Szczypiński P., Dwojakowski G., Strzelecki M., Materka A., Computer simulation of magnetic resonance angiography imaging: Model description and validation, PLoS ONE, 9, 2014, DOI: 10.1371/journal.pone.0093689.

[10] Lee J., Smith N.P., The multi-scale modelling of coronary blood flow, Ann Biomed Eng., 40, 2012, 2399-2413.

[11] Lesage D., Angelini E.D., Bloch I., Funka-Lea G., A review of 3D vessel lumen segmentation techniques: models, features and extraction schemes, Medical image analysis, 13, 2009, 819-845.

[12] Polanczyk A., Podyma M., Stefanczyk L., Szubert W., Zbicinski I., A 3D model of thrombus formation in a stent-graft after implantation in the abdominal aorta, Journal of Biomechanics, 48, 2015, 425-431.

[13] Polanczyk A., Podyma M., Stefanczyk L., Zbicinski I., Effects of stent-graft geometry and blood hematocrit on hemodynamic in Abdominal Aortic Aneurysm, Chemical and Process Engineering, 33, 2012, 53-61.

[14] Polanczyk A., Podyma M., Trebinski L., Chrzastek J., Zbicinski I., Stefanczyk L., A Novel Attempt to Standardize Results of CFD Simulations Basing on Spatial Configuration of Aortic Stent-Grafts, PloS ONE, 2016, http://dx.doi.org/10.1371/journal.pone.0153332.

[15] Sato Y., Nakajima S., Atsumi H., Koller T., Gerig G., Yoshida S., Kikinis R., 3D MultiScale Line Filter for Segmentation and Visualization of Curvilinear Structures in Medical Image, in: Proc. of CVRMed-MRCAS'97, 1997, Lecture Notes in Computer Science, 1205, 213-222.

[16] Strzelecki M., Szczypinski P., Materka A., Kocinski M., Sankowski A., Level-set segmentation of noisy 3D images of numerically simulated blood vessels and vascular trees, in: Proc. of 6th International Symposium on Image and Signal Processing and Analysis, 2009, 742-747.

[17] Tadeusiewicz R., Śmietański J., Acquisition of Medical Images and their Processing, Analysis, Automatic Recognition and Diagnostic Interpretation [in Polish: Pozyskiwanie obrazów medycznych oraz ich przetwarzanie, analiza, automatyczne rozpoznawanie i diagnostyczna interpretacja], Wydawnictwo STN, Kraków, 2011.

[18] Thierfelder J., Sommer K.M., Baumann W.H., Klotz A.B., Meinel E., Strobl F.G., Nikolaou F.F., Reiser K., von Baumgarten M.F., Whole-brain CT perfusion: reliability and reproducibility of volumetric perfusion deficit assessment in patients with acute ischemic stroke, Neuroradiology, 55, 2013, 827-835.

[19] Valencia A., Figueroa H., Rivera R., Bravo E., Sensitivity analysis of fluid structure interaction in a cerebral aneurysm model to wall thickness and elastic modulus, Advances and Applications in Fluid Mechanics, 12, 2012, 49-66.

[20]Waite L.F., Applied Biofluid Mechanics, McGraw-Hill Professional, New York, 2007.

[21] Woźniak T., Strzelecki M., Segmentation of 3D magnetic resonance brain vessel images based on level set approaches, in: Proc. of IEEE SPA 2015, 2015, 56-61. 
[22] Woźniak T., Strzelecki M., Stefańczyk L., Majos A., 3D vascular tree segmentation using a multiscale vesselness function and a level set approach, Biocybernetics and Biomedical Engineering, 37, 2017, 66-77.

This paper is a revised and extended version of work originally presented at the Signal Processing Algorithms, Architectures, Arrangements, and Applications Conference, 21-23 September 2016, Poznan, Poland

Received 30.08.2016, Accepted 19.07.2017 